\title{
Robust Tissue Boundary Detection for Cerebral Cortical Thickness Estimation
}

\author{
Marietta L.J. Scott and Neil A. Thacker \\ University of Manchester, Manchester, UK \\ marietta.scott@manchester.ac.uk \\ http://www.tina-vision.net
}

\begin{abstract}
This paper presents an algorithm for determining regional cerebral grey matter cortical thickness from magnetic resonance scans. In particular, the modification of a gradient-based edge detector into an iso-grey-level boundary detector for reliably determining the low-contrast grey-white matter interface is described and discussed. The reproducibility of the algorithm over 31 gyral regions is assessed using repeat scans of four subjects, and a technique for correcting the misplacement of the grey-white matter boundary is shown to significantly reduce the systematic error on the reproducibility.
\end{abstract}

\section{Introduction}

The determination and characterisation of human cerebral cortical grey matter thickness has enormous potential use in the assessment of the severity and progression of pathology and of the processes of normal brain ageing. Grey matter (GM) volume loss is seen throughout adulthood to old age [1 and increased cortical thinning relative to control subjects has been implicated in various degenerative diseases, such as Alzheimer's disease [2], and Multiple Sclerosis [3].

The highly convoluted folding of the cortex provides two challenges to the estimation of thickness. The first is the problem of measuring a thickness of a curved structure, the second is the determination of the boundaries of the GM ribbon, particularly when the opposing banks of two cortical folds are sufficiently close that there is no intervening cerebro-spinal fluid (CSF) or white matter (WM). Assuming that the boundaries of the GM ribbon have been accurately segmented in 3D, there are various measures of distance between the two surfaces that might be employed. First, if an active shape model algorithm using correspondences (eg. 4]) has been used to create the surfaces, the distance between the corresponding points on the two surfaces can be taken. However, anatomical homology within a group of subjects will not be precise. Alternatively, the minimum distance or distance along the surface normal between a given point on one surface to the opposing surface can be used. Of these two methods, the minimum distance will always produce a shorter average cortical thickness than the surface normal method [4].

Segmentation of the GM from the underlying WM and enveloping CSF on MR images requires knowledge of the expected grey-level intensity values for 
(a)

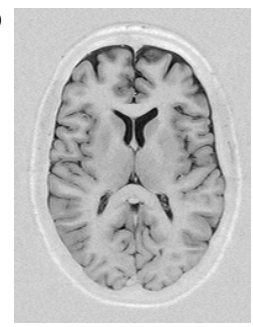

(b)

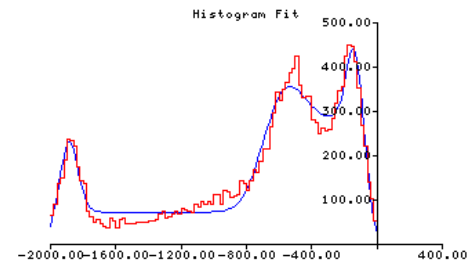

Fig. 1. Example axial inversion-recovery image (a) and corresponding grey-level intensity histogram (b) showing, from left to right, peaks for the CSF, GM and WM

these tissues. The segmentation used must also be able to detect tissue partial voluming and appropriate boundaries should be located under such circumstances. Through-plane magnetic field inhomogeneities should also not be ignored [5]. In addition, the contrast between the tissues needs to be sufficient in order to define their boundaries. Demyelination of WM axons, due to ageing and disease processes will result in the WM appearing more like the non-myelinated GM, making accurate boundary detection less feasible.

The simplest model for boundary determination between two tissues (WM/ GM or GM/CSF) is based upon the assumption of a linear image formation process, such that the boundary occurs at the position where the image grey-level intensity is half-way between the two pure tissue values. As can be seen from fig. 1(b) the contrast at the GM/CSF boundary is large and it should be possible to use an edge detector to determine this boundary, whereas the GM/WM contrast is comparable to the noise in the image, so an edge detector could not be used reliably here. The main emphasis of this paper is to describe a modification to the Canny edge detector [6] for the determination of the GM/WM boundary for subsequent use in an original cortical thickness algorithm. The technique allows an assessment of the accuracy of the boundary positioning, and hence a post-processing correction mechanism for the regional thickness estimates. A reproducibility study is presented, showing that the boundary correction can indeed be used to reduce the systematic error on the cortical thickness measurements.

\section{Materials and Methods}

\subsection{Approach Taken to Determine Cortical Thickness}

The analysis of the data can be divided into two stages; pre-processing to convert the original volume of data into the required form and the actual cortical thickness estimation. Initially, the mean and standard deviation of the grey-level values of the pure tissues (in this case only GM, WM and CSF are considered) in the image volume are determined for use in future processing steps. Greylevel intensity values from a region (comprising several slices in order to average through-plane inhomogeneities to a certain degree) in the frontal lobe representative of the pure tissue values, are histogrammed (as shown in fig. 1) and a 
Bayesian mixture model [7], containing terms for both pure tissues and partial volumes, is fitted to the histogram using simplex to obtain the pure tissue means and standard deviations. To obtain a finer through-plane resolution whilst preserving tissue boundaries, the data is explicitly up-interpolated in the z-direction [8] using a partial volume scheme to constrain the potential tissue boundaries, determined using 3D image gradients, that could pass through a partial volume voxel. The volume of data is then registered to a stereotaxic space (the Talairach atlas [9]) using a linear affine transform. The atlas defines the 31 cortical regions (see table 2 for region names) used later in producing regional histograms of the cortical thickness. Finally, the GM is segmented in the form of the most likely volume estimate in each voxel given the data, based on the defined probability density functions of the image intensity distribution.

The cortical thickness estimation itself proceeds by using a modified edge detection process (see below) to determine the GM/WM boundary. The surface normal to the boundary in 3D at each voxel on the boundary is determined, using a 3D Gaussian smoothed data set (in order to reduce the effects of noise) and a search is performed over $20 \mathrm{~mm}$ in this direction on the segmented GM map until an edge (see [10] for precise details) is found. The cortical thickness at each boundary voxel is inserted into the appropriate regional histogram according to the registration into stereotaxic space determined earlier. The median of each regional histogram is calculated in order to give a robust estimation of the average cortical thickness for each region.

\subsection{Modification to the Canny Edge Detector}

The approach to thickness measurement presented here is inherently feature based and the GM/WM boundary is of particular importance. Conventional edge detectors are particularly poor at identifying edge boundaries where the contrast approaches the noise level, such as found at the GM/WM boundary. However, this boundary is expected to have one consistent grey-level value, in the absence of field inhomogeneities and tissue variation, and in this case is defined as the boundary at the average of the two pure tissue (50\% transition) values. A simple 'z-score' measure of the consistency of the grey-level of each voxel with this boundary midpoint value is used to construct a likelihood image (which highlights the GM/WM boundary) based on a Gaussian distribution with a standard deviation of ten times the noise in the input image. This is monotonically related to a true hypothesis probability, but has better numerical properties for subsequent processing and sub-voxel peak location. This can be used as the basis for the enhancement process rather than the conventional (summed squares) gradient based measure and subsequent stages of the detection process are applied as normal. The implementation is based upon a version of the Canny edge detector. This new iso-contour Canny (or Iso-Canny) algorithm has the advantage that although noise processes may move the position of the detected boundary, these processes cannot prevent detection of a transition.

The later stages of the standard Canny edge detector perform non-maximal suppression and hysteresis thresholding which results in well localised connected 

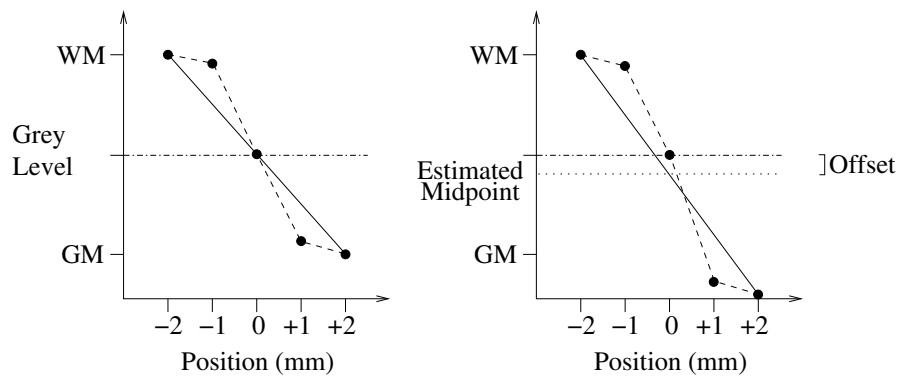

Fig. 2. Diagram illustrating the midpoint offset calculation. The plots show position on the $\mathrm{x}$-axis and grey-level on the $\mathrm{y}$-axis. Marked are the pure tissue grey-level values for GM and WM and the midpoint between them is shown by the dash-dot line. The central position is at the midpoint (edge) and the two positions on each side are in GM and WM. The left-hand plot shows the ideal case, where the linear interpolation (solid line) between the grey-level values of the positions $2 \mathrm{~mm}$ either side corresponds to the midpoint value. The right-hand plot shows an example of the case where the value of the position in the GM is less than the pure tissue value, so that the interpolated grey-level value at the central position is less by some offset than the midpoint (edge) value.

edge strings which persist into low edge strength regions. Edge locations are computed within each slice of the data to sub-pixel accuracy (to $0.1 \mathrm{~mm}$ reproducibility) from quadratic fits to the peaks in the edge strength map. This whole process is particularly reliant on the assumption that one accurately determined GM/WM boundary value is applicable in all parts of the image volume. The following section describes the implementation of a quality control process to assess any systematic errors in the analysis, or failures of the calibration process.

\subsection{Post-processing to Determine the "Correct" Boundary Position}

Use of an incorrect grey-level value for the midpoint of the GM and WM will result in a large systematic error on the regional cortical thickness. Presented here is a technique for monitoring this effect and for correcting for it post hoc if required. In order to calibrate such a correction for a given subject, the effect on the median thickness in each region by perturbing the GM/WM grey-level midpoint by -80 to +80 (in increments of 20 grey-levels) was investigated (using in each case the same set of grey-level and probability images). The regression coefficients of median thickness against grey-level perturbation are presented in section 3.2 A method for determining the difference ("offset") between the pre-determined midpoint grey-level value and the value at the actual GM/WM boundary is presented in fig. 2. In order to obtain an average value for each region, the offsets at each tissue boundary location are entered into regional histograms. The position of the peak of each histogram is taken as the offset estimate for that region. This grey-level offset can be used to compute an equivalent median thickness error, using the appropriate regression coefficients (given in table 2) as determined earlier. 


\subsection{Subjects and Scan Parameters}

4 normal volunteers (all male, ages: 34, 40, 40, 46) underwent MR scans on two occasions within 7-21 days apart. All subjects gave informed consent and the study was approved by the Central Manchester LREC. An axial anatomical inversion recovery MR sequence (eg; fig. 1) was acquired (Philips Medical Systems, Best, The Netherlands, 1.5 Tesla, TI/TR/TE $=300 / 6850 / 18 \mathrm{~ms}, 90^{\circ}$ flip angle, echo train length $=9$, matrix size $=256^{2}$, in-plane resolution $=0.898^{2} \mathrm{~mm}$, slice thickness $=3.0 \mathrm{~mm}, 51$ slices taken covering the whole of the brain). These images were used to determine GM thickness in 31 cortical regions, as described above. Reproducibility of the technique was assessed by comparison of the regional median thicknesses of the two acquisitions from each subject. Images from the subject with the worst reproducibility underwent the correction for the misplacement of the Iso-Canny boundary, and the reproducibility of the modified results was assessed.

\section{Results}

\subsection{Reproducibility Results}

Figure 3 shows the 31 regional cortical thickness estimates from the 2nd scan plotted against those of the first, for all four subjects. Table 1 gives the corresponding regression coefficients, and the standard error on their measurement for the 4 subjects. Subjects 2, 3 and 4 have slopes which lie within $5 \%$ of the expected value of 1 , whereas subject 1 shows a $16 \%$ error. The systematic error on the thickness measurements for this group is calculated as the RMS of the differences between the line of equality and the coefficients, scaled by $\sqrt{2}$ because two measurements have been taken, and amounts to $6.2 \%$ on the measurement in any given individual. The standard error of the data is indicative of the statistical error due to sampling the thickness distribution on any given region of the data. This can be calculated as the product of the standard error and the $\sqrt{N}$ where $\mathrm{N}$ is the number of regions, and amounts to no more than $0.06 \mathrm{~mm}$.

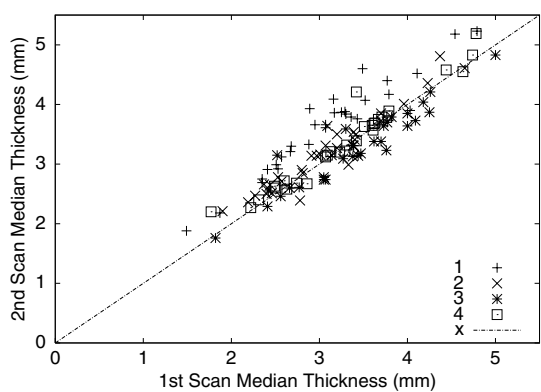

Fig. 3. Scatterplot of 2nd vs 1st scan cortical thickness measurements for 31 regions in each of 4 subjects. The line of equality is also shown.
Table 1. Table of fitted slopes and standard error on the fit for each subject for the data in fig. 2. Note that all regressions are constrained to pass through the origin.

\begin{tabular}{ccc}
\hline Subject & Slope & Std. Err \\
\hline 1 & 1.16 & 0.0148 \\
2 & 1.04 & 0.0111 \\
3 & 0.95 & 0.0098 \\
4 & 1.03 & 0.0088 \\
\hline
\end{tabular}




\subsection{Iso-Canny Correction Results}

Table 2 gives, for the two scans of subject 1, the regression coefficients of the slopes of median thickness against the extent of GM/WM midpoint perturbation, as well as the estimated offset values by which the boundary was misplaced. In the majority of regions, the slopes are near perfect negative linear correlations of

Table 2. Table of the Talairach regions investigated, values of the slopes of median thickness against Iso-Canny midpoint used and calculated offset values for the two scans of subject 1

\begin{tabular}{|c|c|c|c|c|c|}
\hline \multirow[t]{2}{*}{ Lobe } & \multirow[t]{2}{*}{ Region } & \multicolumn{2}{|c|}{$\begin{array}{c}\text { Slope }\left(\times 10^{-3}\right) \\
(\mathrm{mm} / \text { grey-level })\end{array}$} & \multicolumn{2}{|c|}{$\begin{array}{c}\text { Offset } \\
\text { (grey-levels) }\end{array}$} \\
\hline & & Scan 1 & Scan 2 & Scan 1 & Scan 2 \\
\hline \multirow{7}{*}{ Frontal } & Rectal Gyrus & -4.08 & -1.64 & 8.00 & -126.67 \\
\hline & Orbital Gyrus & -1.23 & -1.08 & -173.33 & 127.62 \\
\hline & Precentral Gyrus & -4.64 & -8.26 & -23.33 & -50.13 \\
\hline & Inferior Gyrus & -4.37 & -6.47 & -16.57 & -67.88 \\
\hline & Middle Gyrus & -4.50 & -6.62 & -30.14 & -74.17 \\
\hline & Superior Gyrus & -4.96 & -7.23 & -32.64 & -82.07 \\
\hline & Medial Gyrus & -4.33 & -7.46 & -21.78 & -48.68 \\
\hline \multirow{3}{*}{ Limbic } & Posterior Cingulate & -1.30 & -5.28 & -10.26 & -45.81 \\
\hline & Anterior Cingulate & -6.32 & -7.03 & -7.27 & -47.69 \\
\hline & Subcallosal Gyrus & -2.12 & 2.82 & -126.67 & -60.44 \\
\hline \multirow{5}{*}{ Occipital } & Inferior Gyrus & -2.24 & -4.28 & -3.64 & -46.67 \\
\hline & Lingual Gyrus & -2.03 & -5.03 & -19.39 & -95.24 \\
\hline & Middle Gyrus & -2.48 & -5.09 & 5.22 & -45.46 \\
\hline & Superior Gyrus & -2.04 & -4.82 & -55.24 & -156.00 \\
\hline & Cuneus & -2.67 & -5.61 & -20.98 & -58.24 \\
\hline \multirow{9}{*}{ Parietal } & Insula & -3.15 & -2.53 & -34.02 & -72.94 \\
\hline & Angular Gyrus & -3.93 & -4.89 & -51.43 & -99.26 \\
\hline & Supramarginal Gyrus & -3.43 & -6.10 & -22.22 & -52.08 \\
\hline & Cingulate Gyrus & -3.93 & -5.03 & -18.58 & -54.81 \\
\hline & Inferior Lobule & -4.03 & -5.79 & -20.39 & -88.79 \\
\hline & Superior Lobule & -4.33 & -5.80 & -37.58 & -70.77 \\
\hline & Paracentral Lobule & -3.73 & -7.26 & -110.30 & -108.57 \\
\hline & Postcentral Gyrus & -3.92 & -6.85 & -34.67 & -60.61 \\
\hline & Precuneus & -3.83 & -6.48 & -34.29 & -67.83 \\
\hline \multirow{7}{*}{ Temporal } & Transverse Gyrus & -5.58 & -9.83 & 8.48 & -20.00 \\
\hline & Uncus & -2.23 & -1.37 & -42.67 & -70.83 \\
\hline & Fusiform Gyrus & -1.93 & -4.18 & -21.40 & -65.78 \\
\hline & Inferior Gyrus & -3.07 & -2.88 & -41.90 & -95.76 \\
\hline & Parahippocampal Gyrus & -1.91 & -4.23 & -18.63 & -76.92 \\
\hline & Middle Gyrus & -2.66 & -5.45 & -22.48 & -54.57 \\
\hline & Superior Gyrus & -3.22 & -4.21 & 2.37 & -42.46 \\
\hline
\end{tabular}


thickness with midpoint perturbation. The offset results demonstrate a negative bias (roughly ranging between 0 and -100) which is also seen in a larger cohort (results not shown). This is consistent with the GM grey-levels being lower than expected. The thickness estimation technique assumes that the average of two pure tissue values can be taken as the value at the boundary between these tissues. However, due to the slice thickness of this data, it is likely that there are substantial partial volume effects. GM/CSF partial volumes will result in a more pronounced effect than GM/WM partial volumes, which could explain the peak shift seen in the data If this is the case, the boundary correction may still be used for correcting the systematic error, even though the peak is no longer expected to be at the same grey-level as the assumed tissue boundary.

Applying the Iso-Canny offset correction to the regional cortical thickness measurements from both scans of subject 1 reduces the slope of the regressions from $16 \%$ greater than unity to $9.7 \%$ and marginally improves the standard error on fitting the regressions from 0.0148 to $0.0133 \mathrm{~mm}$.

\section{Discussion}

This paper has presented some of the difficulties in reliably determining GM cortical thickness from images obtained using MRI. Descriptions of a cortical thickness algorithm and the required pre-processing stages are given. The method is applied to repeat scans of four young normals, and the reproducibility of the whole procedure is assessed. The large systematic error, at $6.2 \%$, mainly due to the initial grey-level histogram parameter determination, has implications for the use of this cortical thickness estimation in longitudinal studies. Such an error would be expected to mask any real changes in cortical thickness in an individual over the time scale in which the repeated measurements are likely to be made. However, several steps can be taken in order to achieve greater consistency. Ensuring an identical scanner set-up and subject physiology, as well as fixing the Bayesian prior terms and the ratio of grey-level histogram peaks between scans should reduce variability. Note that natural variation between subjects will be far greater than the effects of the systematic error, such that group-wise comparisons are feasible on tens of subjects. However, the ultimate goal of this work is to provide information suitable for decision support in individual subjects.

The main focus of the paper is a description of a GM/WM boundary detector. The advantage of basing this upon a conventional edge detector is that it provides edge locations computed to sub-pixel accuracy, as is necessary when measuring structures as small as the GM ribbon. In addition, well localised connected edge strings persist even in conventional low edge strength regions. The effect of perturbing the GM/WM midpoint value, in order to determine the effect of modifing the position of the GM/WM edge strings on the regional thickness estimation was used to calibrate a regional correction factor. This was used in conjunction with a technique for determining the inaccuracy in the position of the boundary, for the subject with the greatest systematic error, in order to produce a distance by which the regional thickness was in error. The modified 
regional estimates were then used to assess any change in reproducibility afforded by the boundary correction technique. The technique improved the systematic error in all 31 regions of subject 1 from $16 \%$ to $9 \%$, so the technique appears to be beneficial and implies that through-plane inhomogeneities are partly responsible. However, the error is still greater than that exhibited by the other three subjects, probably for reasons associated with the segmentation. If the tissue value estimations led to inaccuracies in the GM/WM boundary, then the GM/CSF boundary found on the segmented GM maps may also have been inaccurate, although misplacement of the GM/CSF boundary by 50 grey-levels or so will have a much smaller effect on the median thickness than misplacing the GM/WM boundary by the same amount.

\section{Acknowledgements}

This work was supported by a grant from the Wellcome Trust. Thanks to Prof. P. Rabbitt for the subject data and Dr. Paul Bromiley for the segmentation code.

\section{References}

1. Sowell, E.R., Petersen B.S., Thompson P.M. et al: Mapping cortical change across the human life span. Nature Neuroscience 6 (2003) 309-315

2. Double K.L., Halliday G.M., Krill J.J., Harasty J.A. et al: Topography of brain atrophy during normal aging and Alzheimer's disease. Neurobiol. Aging 17 (1996) 513-521

3. Sailer M., Fischl B., Salat D., Tempelmann C. et al: Focal thinning of the cerebral cortex in multiple sclerosis. Brain 126 (2003) 1734-1744

4. MacDonald D., Kabani N., Avis D., Evans A.C.: Automated 3-D extraction of inner and outer surfaces of cerebral cortex from MRI. NeuroImage 12 (2000) 340-356

5. Jones S.E., Buchbinder B.R., Aharon I.: Three-dimensional mapping of cortical thickness using Laplace's equation. Hum. Br. Map. 11 (2000) 12-32

6. Canny J.F.: A Computational Approach to Edge Detection. Patt. Anal. Mach. Intel. 8 (1986) 679-698

7. Pokrić M., Thacker N.A., Scott M.L.J., Jackson A.: Multi-dimensional medical image segmentation with partial voluming. Proc. MIUA 5 (2001) 77-81

8. McKie S., Thacker N.A.: Step interpolation of MR images with inter-slice gap correction. Tina Memo 2003-010 (2003)

9. Talairach J., Tournoux P.: Co-planar stereotaxic atlas of the human brain. 3dimensional proportional system: An approach to cerebral imaging. Thieme medical publishers (1988)

10. Scott M.L.J., Thacker N.A.: Cerebral Cortical Thickness Measurements. Tina Memo 2004-007 (2004) 